\title{
Extraintestinal pathogenic Escherichia coli in Saudi Arabia: A review of antimicrobial resistance and molecular epidemiology
}

\author{
Abdulaziz Alqasim \\ Department of Clinical Laboratory Sciences, College of Applied Medical Sciences, King Saud University, PO Box 10219, \\ Riyadh 11433, Saudi Arabia \\ *For correspondence: Email: aalqasim@ksu.edu.sa
}

Sent for review: 28 October 2019

Revised accepted: 29 January 2020

\begin{abstract}
Extra-intestinal pathogenic Escherichia coli (ExPEC) is commonly associated with causing urinary tract and bloodstream infections. Over the past two decades, the antimicrobial resistance of EXPEC has increasingly been reported [1]. Given that Saudi Arabia annually hosts mass religious events, such as Hajj, this review investigated several aspects of antimicrobial resistance of EXPEC in this country including the current prevalence of resistance and molecular epidemiology of ExPEC isolates. Generally, the overall prevalence of antibiotic resistance of EXPEC in Saudi Arabia is on increase. The current emergence of colistin resistance in EXPEC represents a major challenge to public health. Local molecular epidemiological studies have shown the dominance of E. coli sequence type 131 (E. coli ST131) over other major ExPEC STs. This is an important observation given that this clone has been associated with high multidrug resistance and extended-spectrum $\beta$-lactamases carriage. To reduce the burden of this resistance in the future, it would be crucial to avoid uncontrolled use of antibiotics in either clinical settings or animal food industry.
\end{abstract}

Keywords: Extra-intestinal pathogenic Escherichia coli, Antimicrobial resistance, ST131, Saudi Arabia, Colistin resistance, Extended-spectrum $\beta$-lactamases

This is an Open Access article that uses a fund-ing model which does not charge readers or their institutions for access and distributed under the terms of the Creative Commons Attribution License (http://creativecommons.org/licenses/by/4.0) and the Budapest Open Access Initiative (http://www.budapestopenaccessinitiative.org/read), which permit unrestricted use, distribution, and reproduction in any medium, provided the original work is properly credited.

Tropical Journal of Pharmaceutical Research is indexed by Science Citation Index (SciSearch), Scopus, International Pharmaceutical Abstract, Chemical Abstracts, Embase, Index Copernicus, EBSCO, African Index Medicus, JournalSeek, Journal Citation Reports/Science Edition, Directory of Open Access Journals (DOAJ), African Journal Online, Bioline International, Open-J-Gate and Pharmacy Abstracts

\section{INTRODUCTION}

Extra-intestinal pathogenic Escherichia coli (ExPEC) is associated with causing urinary tract infections (UTIs), bloodstream infections (BSIs), neonatal meningitis and pneumonia [2].

In the recent years, the level of antibiotic resistance of E. coli, particularly ExPEC, to firstline agents such as ciprofloxacin and cephalosporins, has increased markedly [1]. Furthermore, this resistance has further increased globally to include many powerful antibiotic families such as polymyxins [3].

The Kingdom of Saudi Arabia is the annual host of mass religious events, known as Umrah and Hajj. These events are associated with high risk of infectious disease transmission [4] which makes the kingdom a potential center for the 
spread of antimicrobial resistant bacterial strains around the world.

This review aimed to summarize the current knowledge of antimicrobial resistance of ExPEC in Saudi Arabia, focusing on the overall local prevalence of antimicrobial resistance and molecular epidemiology of ExPEC isolates. The PubMed database was used to search the literature from 1987 to 2018, with the following phrases: Escherichia coli, extra-intestinal pathogenic E. coli, ESBL-producing E. coli, molecular epidemiology of extra-intestinal pathogenic E. coli, Saudi Arabia, antibiotics and antimicrobial resistance. Reference lists of relevant research articles were searched for more information.

\section{Current prevalence of antimicrobial resistance of ExPEC in Saudi Arabia}

In this section, the current local prevalence of antimicrobial resistance of ExPEC isolates, to major antibiotic classes is demonstrated.

\section{Resistance to $\beta$-lactams}

\section{Penicillins/penicillin-beta-lactamase inhibitor combinations}

Early local reports showed that $E$. coli resistance to penicillins, such as amoxicillin and ampicillin, was reported in more than $50 \%$ of isolates $[5,6]$. Nonetheless, penicillin-beta-lactamase inhibitor combinations showed better activity against $E$. coli compared to penicillins [7] (Table 1). Recently, the spectrum of ExPEC resistance to penicillins has increased significantly. For instance, it has been demonstrated that all UPEC isolates were resistant to ampicillin [8], and that 85 and $100 \%$ of UPEC isolates were resistant to piperacillin and amoxicillin, respectively [9]. Although ExPEC resistance to penicillin-betalactamase inhibitor combination agents, particularly piperacillin/tazobactam, has recently been found to be low [10, 11], Al-Agamy and colleagues have shown that resistance to amoxicillin/clavulanic acid and piperacillin/tazobactam was found in 96 and 50 $\%$ of the tested UPEC isolates, respectively [9].

The current high local resistance levels to penicillins and penicillin-beta-lactamase inhibitor combinations among ExPEC isolates have strongly been associated with ESBL carriage. It has been found that ESBL-producing ExPEC isolates were completely resistant to ampicillin, pipracillin and amoxicillin/clavulanic acid [9]. Resistance to piperacillin/tazobactam was also high among ESBL-producing ExPEC isolates, with $77.4 \%$ of these isolates showing resistance to this agent [9] (Table 1).

\section{Cephalosporins}

Previous data demonstrated that about $70 \%$ of all $E$. coli isolates were susceptible to firstgeneration cephalosporins including cefazolin [6]. However, it was found that cefalotin was associated with low activity against $E$. coli $[7,12]$. $E$. coli resistance to second-generation cephalosporins such as cefoxitin and cefuroxime, was found to be significantly variable between agents, with a relatively low resistance to cefoxitin (range: 5 - $7 \%$ ) [6] when compared to much higher resistance to cefuroxime (range: 13 - $56 \%$ ) [6,13]. Furthermore, the range of $E$. coli resistance to third-generation cephalosporins was between 6 and $49 \%$ of all $E$. coli isolates $[6,9,10,12,13]$, while it was between 2 and $54 \%$ of all $E$. coli isolates for fourth-generation cephalosporins $[9,10,12]$.

Recently, the level of ExPEC resistance to cephalosporins has increased markedly. For example, Al-Mijalli has reported that about $99 \%$ of the all tested $E$. coli isolates were resistant to cefalotin, ceftriaxone, cefuroxime and ceftazidime, while resistance to cefoxitin and cefepime was reported in 14 and $96.7 \%$ of isolates, respectively [14]. Another report has demonstrated that ESBL-producing UPEC isolates were associated with complete resistance to cefotaxime, ceftazidime and cefepime, and it also showed that $70.3 \%$ of these isolates were cefoxitin-resistant [9] (Table 1).

\section{Monobactams}

Previous local reports showed that the range of E. coli resistance to monobactams such as aztreonam, was between 36 and 63\% [6,10,13]. However, the level of ExPEC resistance to aztreonam has recently increased. For instance, it has been found that $74.4 \%$ of ESBL-producing UPEC isolates were aztreonam-resistant [14] (Table 1). Another study has demonstrated that all tested ESBL-producing UPEC isolates were aztreonam-resistant, while aztreonam resistance remained very low among non-ESBL-producing ExPEC isolates and it was only detected in $1.65 \%$ of the tested isolates [9].

\section{Carbapenems}

Over the past decade, E. coli resistance to carbapenems, such as imipenem and meropenem, has been uncommon, and the range of resistance was $0-8.5 \%$ for imipenem and $0-1.1 \%$ for meropenem $[6,14,15]$. 
Currently, although the majority of recently published reports have concluded that ExPEC isolates remained totally susceptible to imipenem and meropenem $[9,16]$, AlYousef and others have demonstrated that 15 and $22.5 \%$ of UPEC isolates were resistant to imipenem and ertapenem, respectively [8] (Table 1). This finding is important given that carbapenems are used for treating serious infections caused by multidrug resistant (MDR) ExPEC isolates, and such resistance makes the management of patients more complicated.

\section{Resistance to aminoglycosides}

Until early 2000s, the local prevalence of $E$. coli resistance to aminoglycosides was low; with less than $10 \%$ of $E$. coli isolates showing resistance to aminoglycosides $[5,6,8,10,17]$. However, a substantial increase in the level of resistance of ExPEC to some aminoglycosides agents, particularly amikacin and tobramycin, has been reported. For example, it has been found that $78 \%$ of ExPEC isolates were resistant to amikacin, $47.8 \%$ were non-susceptible to gentamicin while $56.5 \%$ were resistant to tobramycin [18] (Table 1).

\section{Resistance to fluoroquinolones}

Early local studies reported high E. coli resistance to fluoroquinolones, such as ciprofloxacin, norfloxacin and levofloxacin. For example, resistance to ciprofloxacin ranged between 25.8 and $51 \%$ [6,11], while resistance to norfloxacin was observed in $10 \%$ of $E$. coli isolates [6]. More recent studies have shown higher levels of fluoroquinolone resistance in ExPEC isolates than was reported previously. For instance, Al Mously and others have detected ciprofloxacin resistance in about $70 \%$ of all tested ExPEC isolates [15], while Alyamani and co-authors have demonstrated that about $80 \%$ of ExPEC isolates were ciprofloxacinresistant [19]. Norfloxacin resistance of ExPEC has also recently increased, and this resistance has been reported in as many as $44 \%$ of ExPEC isolates [10]. ExPEC resistance to levofloxacin has also been high, ranging between 28 and 63 $\%[8,11,14]$ (Table 1).

\section{Resistance to colistin}

Globally, E. coli resistance to colistin has recently been reported [3]. Until 2016, E. coli resistance to colistin was not reported locally. Although the majority of past local studies did not include colistin in the antibiotic panels used to determine the antibiotic susceptibility profiles of E. coli, a previous study showed that all tested
ExPEC isolates were completely susceptible to colistin [9]. However, Sonnevend and others described the first case of colistin resistance among ExPEC isolates in 2016, and they found a colistin resistant ExPEC blood isolate harboring the gene conferring resistance to colistin, namely mcr-1 [20]. This finding is of great importance given that colistin is currently used to treat many MDR ExPEC isolates that show resistance to powerful antibiotic families e.g. carbapenems, and such resistance might lead to the emergence and dissemination of pandrug resistant (PDR) ExPEC isolates in the future.

\section{Resistance to other antibiotics}

Many previous reports showed that $E$. coli resistance to nitrofurantoin has been very low, with more than $90 \%$ of isolates showing susceptibility to this agent $[6,15]$. More recently, the prevalence of nitrofurantoin resistance among UPEC isolates has increased, and this resistance was observed in as high as $15 \%$ of UPEC isolates [14] (Table 1).

Before 2010, E. coli resistance to tetracycline ranged between 50 and $54.5 \%$ of total $E$. coli isolates [6]. More recently, the spectrum of ExPEC resistance to tetracycline has increasingly been reported, and this resistance has been found in as high as $65.2 \%$ of ExPEC isolates [18]. Tigecycline is highly active against E. coli and a previous report found a complete susceptibility of $E$. coli isolates to this agent [13]. However, higher tigecycline resistance has recently been detected locally, with $13 \%$ of isolates showing resistance to tigecycline [18] (Table 1).

According to many previous reports, the local prevalence of $E$. coli resistance to sulfamethoxazole-trimethoprim ranged between 40 and $66 \%$ of $E$. coli isolates [13, 17] (Table 1). With regard to ExPEC resistance to fosfomycin, Al-Agamy and others have found full susceptibility to fosfomycin among a collection of UPEC isolates [9]. Interestingly, by contrast, AlYousef and colleagues have recently demonstrated that $37.5 \%$ of ExPEC isolates were resistant to fosfomycin [8] (Table 1).

The role of extended-spectrum $\beta$-lactamases carriage in increasing local antimicrobial resistance of EXPEC

It has been shown that the current high rates of resistance to $\beta$-lactam antibiotics are attributed to the worldwide dissemination of extendedspectrum $\beta$-lactamases (ESBLs), particularly among ExPEC [16]. 
Table 1: Reported antimicrobial resistance patterns of ExPEC isolates from Saudi Arabia

\begin{tabular}{|c|c|c|c|c|c|c|c|c|}
\hline \multirow[t]{2}{*}{ Agent } & \multirow{2}{*}{$\begin{array}{l}\text { Resistance } \\
\text { Range (\%) } \\
\end{array}$} & \multicolumn{7}{|c|}{ Number of studies reporting resistance rates within the range ${ }^{\text {Ret }}$} \\
\hline & & $0 \%$ & $\leq 10 \%$ & $>10-25 \%$ & $>25-50 \%$ & $>50-75 \%$ & $>75 \%$ & $100 \%$ \\
\hline \multicolumn{9}{|c|}{ Penicillins/combinations } \\
\hline Amoxicillin & $61-100$ & & & & & & $1^{[1 y]}$ & $1^{[y]}$ \\
\hline Ampicillin & $60-100$ & & & & & $2^{\lfloor b, b\rfloor}$ & $2^{[10,13]}$ & $1^{[8]}$ \\
\hline Pipracillin & $33-100$ & & & & & $2^{[b, 6]}$ & $1^{[y]}$ & $1^{[y]}$ \\
\hline $\begin{array}{l}\text { Amoxicillin/ } \\
\text { clavulanic acid }\end{array}$ & $20-96$ & & & $2^{[/, 11]}$ & $1^{[13]}$ & $2^{[8,10]}$ & $1^{[y]}$ & \\
\hline $\begin{array}{l}\text { Pipracillin/ } \\
\text { tazobactam }\end{array}$ & $0-50$ & $1^{[10]}$ & & $1^{[8]}$ & $1^{[y]}$ & & & \\
\hline \multicolumn{9}{|l|}{ Cephalosporins } \\
\hline Cefazolin & $19-77.5$ & & & $1^{\lfloor b\rfloor}$ & $1^{[10]}$ & & $1^{[8]}$ & \\
\hline Cefalotin & $48-98.9$ & & & & $1^{[12]}$ & $1^{[/]}$ & $2^{\lfloor 10,14]}$ & \\
\hline Cefoxitin & $5-71.7$ & & $1^{\lfloor b]}$ & $1^{[14]}$ & $1^{[8]}$ & $1^{[y]}$ & & \\
\hline Cefuroxime & $13-98.9$ & & & $1^{[b]}$ & $1^{[10]}$ & $1^{[13]}$ & $1^{[14]}$ & \\
\hline Ceftriaxone & $6-98.9$ & & $2^{[6,12\rfloor}$ & & $1^{\lfloor 13]}$ & & $1^{[14]}$ & \\
\hline Cefotaxime & $6-100$ & & $1^{[12]}$ & $1^{[y]}$ & $1^{[10]}$ & $1^{[8]}$ & $1^{[19]}$ & $1^{[y]}$ \\
\hline Ceftazidime & $6-100$ & & $1^{[12]}$ & $1^{[b]}$ & $2^{[10,13\rfloor}$ & & $1^{[14]}$ & $1^{[y]}$ \\
\hline $\begin{array}{l}\text { Cefepime } \\
\text { Monobactams }\end{array}$ & $2-100$ & & $1^{[12]}$ & $1^{[y]}$ & $1^{[10]}$ & $1^{[13]}$ & $1^{[14]}$ & $1^{[y]}$ \\
\hline Aztreonam & 21.1-98.9 & & & $1^{[y]}$ & $2^{[10,13]}$ & $1^{[6]}$ & $1^{[14]}$ & \\
\hline Aminoglycosides & & & & & & & & \\
\hline Gentamicin & $1.6-48.4$ & & $2^{\lfloor b, 6]}$ & $1^{[11]}$ & $3^{[8,10,13]}$ & & & \\
\hline Tobramycin & $10-62.5$ & & $1^{[1 /]}$ & $2^{\lfloor 6,11]}$ & & $1^{[18]}$ & & \\
\hline Amikacin & $0-78.3$ & $1^{[1 /]}$ & $2^{[10,73]}$ & & $1^{[y]}$ & & $1^{[18]}$ & \\
\hline \multicolumn{9}{|l|}{ Sulphonamides } \\
\hline $\begin{array}{l}\text { Sulfamethoxazole- } \\
\text { trimethoprim }\end{array}$ & $40-66$ & & & & $1^{[1 /]}$ & $1^{[13]}$ & & \\
\hline \multicolumn{9}{|l|}{ Carbapenems } \\
\hline Imipenem & $0-15$ & $2^{[y, 16]}$ & $3^{\lfloor 6,14,15]}$ & $1^{[8]}$ & & & & \\
\hline Meropenem & $0-1.1$ & $2^{[y, 16]}$ & $2^{[6,14\rfloor}$ & & & & & \\
\hline Ertapenem & $1.1-22.5$ & & $1^{\lfloor 14]}$ & $1^{[8]}$ & & & & \\
\hline \multicolumn{9}{|l|}{ Nitrofurans } \\
\hline Nitrofurantoin & $3-37.3$ & & $2^{\lfloor 6,15\rfloor}$ & $1^{[14]}$ & & & & \\
\hline Fluoroquinolones & & & & & & & & \\
\hline Ciprofloxacin & $25.8-79.7$ & & & & $2^{\lfloor 6,11]}$ & $1^{[15]}$ & $1^{[1 y]}$ & \\
\hline Levofloxacin & 28-63.2 & & & & $2^{[8,11]}$ & $1^{[14]}$ & & \\
\hline $\begin{array}{l}\text { Norfloxacin } \\
\text { Tetracyclines }\end{array}$ & $10.5-44$ & & & $1^{[6]}$ & $1^{\lfloor 10]}$ & & & \\
\hline Tetracycline & $49-65.2$ & & & & $1^{[6]}$ & $1^{[18]}$ & & \\
\hline Tigecycline & $0-48$ & $2^{\lfloor y, 13]}$ & $1^{[14]}$ & $1^{[18]}$ & & & & \\
\hline Fosfomycins & & & & & & & & \\
\hline $\begin{array}{l}\text { Fosfomycin } \\
\text { Polymyxins }\end{array}$ & $0-37.5$ & $1^{[y]}$ & $1^{\lfloor 18]}$ & & $1^{[8]}$ & & & \\
\hline Colistin & 0 & $2^{\lfloor y, 13\rfloor}$ & & & & & & \\
\hline
\end{tabular}

With regard to the prevalence of ESBL-producing ExPEC in Saudi Arabia, a previous study showed that the prevalence of ESBL-production among ExPEC isolates was $6.5 \%$ in 2002 [21]. Recently, this prevalence has increased markedly, ranging between 20.4 and $41.9 \%$ of ExPEC isolates $[8,9,15,16]$ (Table 2).

Molecular characterization of ESBL genotypes among ExPEC isolates has shown that bla mgenes, encoding for CTX-M enzymes, were highly prevalent when compared to the traditional $\beta$-lactamases encoding genes such as bla $a_{T E M}$, bla $a_{S H V}$ and bla $a_{O X A}$, and that bla $a_{C T X-M-15}$ was the most common CTX-M variant $[9,16]$ (Table 2).
Interestingly, the spread of CTX-M-15 enzyme has been strongly associated with high levels of resistance to penicillins, cephalosporins, aminoglycosides, nitrofurans and fluoroquinolones among EXPEC isolates $[9,16]$. These findings highlight the major role of ESBL carriage in driving the high resistance levels among ExPEC isolates.

\section{Molecular epidemiology of MDR ExPEC in Saudi Arabia}

In Saudi Arabia, multilocus sequence typing (MLST)-based studies have generally shown considerable clonal diversity within ExPEC 
populations [19, 22-24]. However, E. coli ST131 was the most common ST type among the tested ExPEC isolates [19, 22, 24]. This section focuses on the most common ExPEC STs circulating locally.

\section{E. coli ST131 complex}

Recent studies have demonstrated that $E$. coli ST131 was the predominant ST type among all ExPEC STs in Saudi Arabia (Table 3) $[19,22,24,25]$. The local prevalence of ST131 isolates ranged between 17.3 and $61.7 \%$ of all ExPEC isolates. Alqasim and co-authors have found that ST131 accounted for $61.7 \%$ of all tested isolates, that $\mathrm{H} 30$ was the most frequent ST131 sub-clone, and that $\mathrm{H} 30$ isolates were higher than other ST131 sub-clones in terms of ESBL carriage and fluoroquinolone resistance [25]. Alghoribi and others have shown that this clone was highly assoacited with ESBL production and antimicrobial resistance compared to other ExPEC clones [22]. Abd El Ghany et al have characterized 10 carbapenem resistant ExPEC isolates recovered from bacteremia, and identified the presence of one $E$. coli ST131 isolate harboring the New Delhi metallo- $\beta$-lactamase gene, bla NDM-1 $_{\text {[23]. }}$ [2.

\section{E. coli ST73 complex}

In Saudi Arabia, Alghoribi et al have demonstrated that E. coli ST73 was the second most dominant ExPEC ST, accounting for $11.4 \%$ of the total isolates identified in the study [22] (Table 3). However, Alyamani et al have demonstrated that E. coli ST73 was less commonly identified among the tested urine isolates, with only 1 of $58(1.7 \%)$ isolates belonging to this clone [19].

\section{E. coli ST69 complex}

Locally, a previous study found that E. coli ST69 isolates accounted for $7.4 \%$ of the total STs identified in the study [22] (Table 3). However, another report did not identify $E$. coli ST69 in the
UTI strain collection [19]. Abd El Ghany et al found one E. coli ST69 isolate of ten ExPEC bacteremia isolates harboring the carbapenem resistance determinant, bla $a_{N D M-1}[23]$.

\section{E. coli ST95 complex}

In Saudi Arabia, Alghoribi et al have found that $E$. coli ST95 accounted for $5.4 \%$ of the total isolates identified in the study [22]. However, two recent studies did not identify this $S T$ in their strain collections $[19,23]$.

\section{E. coli ST10 complex}

Genotyping studies in Saudi Arabia have identified members of ST10 complex in their strain collections. For example, Alyamani et al have identified 7 of $58(12 \%) \quad E$. coli urine isolates belonging to $E$. coli ST10 complex [19]. Another study has found that ST10 isolates accounted for $6.4 \%$ of the total tested ExPEC isolates [22] (Table 3).

\section{Other clonal ExPEC STs}

Local studies have identified a number of other STs that are variable in terms of antimicrobial resistance levels and ESBL carriage. For instance, a study has shown that E. coli ST38 accounted for $7.4 \%$ of the total isolates, and this ST was highly associated MDR phenotype and high CTX-M carriage [22]. Alyamani et al have also found that $6.9 \%$ of all tested E. coli urine isolates belonged to E. coli ST38 [19].

The MDR E. coli clone ST405 was identified among a collection of UPEC isolates, accounting for about $3 \%$ of the total isolates [22]. Abd El Ghany et al has recently identified 8 out of 10 carbapenem resistant $E$. coli bacteremia isolates belonging to two ST complexes: ST448 complex and ST23 complex [23] (Table 3). ST448 isolates were associated with carrying the carbapenem resistance gene, bla OXA-181, while the ST23 isolates harbored the carbapenem resistance determinant, $b_{\text {NDM-5. }}$.

Table 2: The prevalence of ESBL carriage and common ESBL genotypes among ExPEC isolates from Saudi Arabia

\begin{tabular}{|c|c|c|c|c|c|}
\hline $\begin{array}{l}\text { Year of } \\
\text { sampling }\end{array}$ & Sample source & $\begin{array}{l}\text { Number of } \\
\text { ExPEC } \\
\text { isolates }\end{array}$ & $\begin{array}{l}\text { Number of ESBL- } \\
\text { producing ExPEC } \\
\text { isolates }(\%)\end{array}$ & $\begin{array}{l}\text { Common ESBL } \\
\text { genotypes }\end{array}$ & Reference \\
\hline $2002-2003$ & Urine & 1116 & $72(6.5 \%)$ & $\mathrm{NT}^{1}$ & [21] \\
\hline 2010-2011 & Urine & 152 & $31(20.4 \%)$ & $\begin{array}{c}\text { CTX-M-15, TEM-1 \& } \\
\text { CTX-M-27 }\end{array}$ & [9] \\
\hline 2011-2012 & Urine & 3967 & 1086 (27.4\%) & NT & [15] \\
\hline 2014-2015 & Urine & 520 & $218(41.9 \%)$ & NT & [8] \\
\hline 2018 & Urine & 100 & $33(33 \%)$ & $\begin{array}{c}\text { CTX-M-15, OXA \& } \\
\text { TEM }\end{array}$ & {$[16]$} \\
\hline
\end{tabular}


Table 3: Summary of MLST analysis of ExPEC isolates obtained from population-based studies in Saudi Arabia

\begin{tabular}{|c|c|c|c|c|c|c|}
\hline $\begin{array}{l}\text { Year of } \\
\text { sampling }\end{array}$ & Sample source & $\begin{array}{c}\text { Number of } \\
\text { ExPEC } \\
\text { isolates }\end{array}$ & $\begin{array}{l}\text { Number } \\
\text { of unique } \\
\text { STs }\end{array}$ & $\begin{array}{c}\text { Predominant } \\
\text { ST(s) }\end{array}$ & Other major STs & Reference \\
\hline $\begin{array}{l}2011- \\
2012\end{array}$ & Urine & 202 & 51 & ST131 & $\begin{array}{c}\text { ST73, ST38, } \\
\text { ST69, ST10, } \\
\text { ST127 \& ST95 }\end{array}$ & [22] \\
\hline $\begin{array}{l}2014- \\
2015\end{array}$ & Urine & 58 & 10 & ST131 & $\begin{array}{c}\text { ST10, ST38 \& } \\
\text { ST648 }\end{array}$ & [19] \\
\hline $\begin{array}{l}2014- \\
2015\end{array}$ & $\begin{array}{l}\text { Urine, blood and } \\
\text { wound swabs }\end{array}$ & 80 & 32 & ST131 & ST38 \& ST648 & [24] \\
\hline $\begin{array}{l}2014- \\
2015\end{array}$ & Urine & 10 & 4 & ST448 \& ST23 & ST131 \& ST69 & [23] \\
\hline
\end{tabular}

\section{CONCLUDING REMARKS}

This review has shown that the local prevalence of antimicrobial resistance of ExPEC to many front-line antibiotics has increased drastically in the recent years. More importantly, ExPEC isolates have currently developed resistance to the last-line antibiotics such as carbapenems and polymyxins.

The current high antimicrobial resistance of ExPEC can be attributed to many factors. Firstly, the dominance of MDR ExPEC clones, particularly ST131, has recently been identified locally. This is alarming given that this clone is highly associated with resistance to many firstline agents such as fluoroquinolones and third generation cephalosporins, making the treatment of patients more complicated. In the future, it would be important to launch rapid diagnostic tests to identify these clones in order to reduce the burden of resistance.

Unoptimized use of antibiotics has also resulted in high antimicrobial resistance locally Therefore, monitoring the use of antibiotics in clinical settings or animal food industry is essential to combat resistance issue.

\section{DECLARATIONS}

\section{Conflict of interest}

No conflict of interest is associated with this work.

\section{Contribution of authors}

I declare that this work was done by the author named in this article and all liabilities pertaining to claims relating to the content of this article will be borne by the author.

\section{Open Access}

This is an Open Access article that uses a funding model which does not charge readers or their institutions for access and distributed under the terms of the Creative Commons Attribution License (http://creativecommons.org/licenses/by/ 4.0) and the Budapest Open Access Initiative (http://www.budapestopenaccessinitiative.org/rea d), which permit unrestricted use, distribution, and reproduction in any medium, provided the original work is properly credited.

\section{REFERENCES}

1. Lautenbach E, Patel JB, Bilker WB, Edelstein PH, Fishman NO. Extended-spectrum $\beta$-lactamaseproducing Escherichia coli and Klebsiella pneumoniae: risk factors for infection and impact of resistance on outcomes. Clin Infect Dis. 2001; 32(8): 1162-1171.

2. Smith JL, Fratamico PM, Gunther NW. Extraintestinal pathogenic Escherichia coli. Foodborne Pathog Dis. 2007; 4(2): 134-163.

3. Payne M, Croxen MA, Lee TD, Mayson B, Champagne $S$, Leung V, Bariso S, Hoang L, Lowe C. mcr-1-Positive Colistin-Resistant Escherichia coli in Traveler Returning to Canada from China. Emerg Infect Dis. 2016; 22(9): 1673-1675.

4. Memish ZA, Stephens GM, Steffen R, Ahmed QA. Emergence of medicine for mass gatherings: lessons from the Hajj. Lancet Infect Dis. 2012; 12(1): 56-65.

5. Qadri SH, Ostrawski S, Johnson S, Flournoy $D$. Differences in antimicrobial susceptibilities of clinical isolates in Saudi Arabia and the United States. J Natl Med Assoc. 1987; 79(4): 433-437. 
6. Al-Tawfiq JA. Increasing antibiotic resistance among isolates of Escherichia coli recovered from inpatients and outpatients in a Saudi Arabian hospital. Infect Control Hosp Epidemiol. 2006; 27(7): 748-753.

7. Abo-Shadi MA, Al-Johani AA, Bahashwan AA. Antimicrobial resistance in pathogens causing pediatrics bloodstream infections in a Saudi Hospital. Br Microbiol Res J. 2012; 2(4): 212-227.

8. Al Yousef SA, Younis S, Farrag E, Moussa HS, Bayoumi FS, Ali AM. Clinical and laboratory profile of urinary tract infections associated with extended spectrum $\beta$ lactamase producing Escherichia coli and Klebsiella pneumoniae. Ann Clin Lab Sci. 2016; 46(4): 393-400.

9. Al-Agamy $M H$, Shibl AM, Hafez MM, Al-Ahdal MN, Memish ZA, Khubnani $H$. Molecular characteristics of extended-spectrum $\beta$-lactamase-producing Escherichia coli in Riyadh: emergence of CTX-M-15-producing $E$. coli ST131. Ann Clin Microbiol Antimicrob. 2014; 13(1): 1-7.

10. Haseeb A, Faidah HS, Bakhsh AR, Al Malki WH, Elrggal ME, Saleem F, ur Rahman S, Khan TM, Hassali MA. Antimicrobial resistance among pilgrims: a retrospective study from two hospitals in Makkah, Saudi Arabia. Int $J$ Infect Dis. 2016; 47: 92-94.

11. Balkhi B, Mansy W, Alghadeer S, Alnuaim A, Alshehri A, Somily A. Antimicrobial susceptibility of microorganisms causing Urinary Tract Infections in Saudi Arabia. J Infect Dev Ctries. 2018; 12(4): 220-227.

12. Qadri SH, Cunha BA, Ueno $Y$, Abumustafa $F$, Imambaccus $H$, Tullo $D D$, Domenico P. Activity of cefepime against nosocomial blood culture isolates. $J$ Antimicrob Chemother. 1995; 36(3): 531-536.

13. Saeed NK, Kambal AM, El-Khizzi NA. Antimicrobialresistant bacteria in a general intensive care unit in Saudi Arabia. Saudi Med J. 2010; 31(12): 1341-1349.

14. Al-Mijalli S. Bacterial uropathogens in urinary tract infection and antibiotic susceptibility pattern in Riyadh Hospital, Saudi Arabia. Cell Mol Med. 2017; 3(1): 1-6.

15. Al Mously N, Al Arfaj O, Al Fadhil L, Mukaddam S. Antimicrobial susceptibility patterns of ESBL Escherichia coli isolated from community and hospital-acquired urinary tract infections. J Health Spec. 2016; 4(2): 133139.

16. Alqasim A, Abu Jaffal A, Alyousef AA. Prevalence of Multidrug Resistance and Extended-Spectrum $\beta$ Lactamase Carriage of Clinical Uropathogenic
Escherichia coli Isolates in Riyadh, Saudi Arabia. Int J Microbiol. 2018; 2018: 1-9.

17. Qadri S, Rizvi W, Rahman S, Al-Dayel F, Flournoy D. Saudi Arabian-American differences in antimicrobial resistance of Escherichia, Klebsiella, and Pseudomonas. J Natl Med Assoc. 1989; 81(10): 10611064.

18. Al Yousef SA. Surveillance of Antibiotic-Resistant Bacteria in King Khalid Hospital, Hafr Al-Batin, Saudi Arabia, During 2013. Jundishapur J Microbiol. 2016; 9(9): 1-7.

19. Alyamani EJ, Khiyami AM, Booq RY, Majrashi MA, Bahwerth FS, Rechkina E. The occurrence of ESBLproducing Escherichia coli carrying aminoglycoside resistance genes in urinary tract infections in Saudi Arabia. Ann Clin Microbiol Antimicrob. 2017; 16(1): 1-13.

20. Sonnevend A, Ghazawi A, Alqahtani M, Shibl A, Jamal $W$, Hashmey R, Pal T. Plasmid-mediated colistin resistance in Escherichia coli from the Arabian Peninsula. Int J Infect Dis. 2016; 50: 85-90.

21. Kader AA, Kumar A, Dass SM. Antimicrobial resistance patterns of gram-negative bacteria isolated from urine cultures at a general hospital. Saudi J Kidney Dis Transpl. 2004; 15(2): 135-139.

22. Alghoribi MF, Gibreel TM, Farnham G, Al Johani SM, Balkhy HH, Upton M. Antibiotic-resistant ST38, ST131 and ST405 strains are the leading uropathogenic Escherichia coli clones in Riyadh, Saudi Arabia. J Antimicrob Chemother. 2015; 70: 2757-2762.

23. Abd El Ghany M, Sharaf $H$, Al-agamy MH, Shibl A, HillCawthorne GA, Hong P. Genomic characterization of NDM-1 and 5, and OXA-181 carbapenemases in uropathogenic Escherichia coli isolates from Riyadh, Saudi Arabia. PLoS ONE. 2018; 13(8): 1-16.

24. Yasir M, Ajlan AM, Shakil S, Jiman-Fatani AA, Almasaudi $S B$, Farman M, Baazeem ZM, Baabdullah R, Alawi M, Al-Abdullah N. Molecular characterization, antimicrobial resistance and clinico-bioinformatics approaches to address the problem of extended-spectrum $\beta$ lactamase-producing Escherichia coli in western Saudi Arabia. Sci Rep. 2018; 8(1): 1-11.

25. Alqasim A, Jaffal AA, Alyousef AA. Prevalence and molecular characteristics of sequence type 131 clone among clinical uropathogenic Escherichia coli isolates in Riyadh, Saudi Arabia. Saudi J Biol Sci. 2020; 27(1): 296-302. 